\title{
The Design of Embedded AeroEngine Measurement and Test System Based on ARM and FPGA \\ Yundong Sha ${ }^{1, a}$, Jia Han ${ }^{2, b}$ and Fengtong Zhao ${ }^{1}$
}

${ }^{1}$ Shenyang Aerospace University, Liaoning Key Laboratory of Digital Technology Simulation and Test Techniques, Liaoning, Shenyang, 110136, P.R. China

${ }^{2}$ Shenyang Aerospace University, School of Aeroengine and Energy Engineering, Liaoning, Shenyang, 110136, P.R. China

aydsha2003@vip.sina.com, ${ }^{\mathrm{b}}$ hanjia27@126.com

\section{Keyword: Aeroengine Test System, ARM; FPGA, Linux, Embedded system}

\begin{abstract}
Present Measurement and test system for aeroengine rig testing is primarily based on DSP and PC104 industrial computer, which have the disadvantages of large volume, high power consumption, single function and less-generic. So it is a valuable job to promote the performance of the system employing the latest methods of collaborative design and embedded hardware and software technology.

For designing of the new type improved aeroengine test system, a hardware and software co-design strategy is employed. After a thorough trade-off analysis and comparison among different hardware and software systems, an embedded aeroengine test system based on ARM and FPGA is designed, by using S3C2440A CPU with ARM920T core and FPGA from Xilinx as fundamental hardware data acquisition and process platform, using Linux as real time operating system. The data transmission between ARM and FPGA take the path of dual-port RAM, during which the read-write operation is coordinated by means of interruption. The system uses U-Boot to initialize hardware and load operating system. FPGA is used as foreside of data acquisition system, which sampling parameter setting is loaded by reading configuration file during start-up and read into inner register by SPI bus. Data is exported from flash-chip of test system by means of U-disk or network, and analyzed in PC equipped with specialized software. Strong post-proceeding capacity for multi parameters of aeroengine rig testing can be realized, especially for dynamic signal analysis in time domain, frequency domain.

Because of the use of the new platform, a big promotion has been achieved. I) the capacity of data processing increases by $15 \%$. II) The new system allows numbers of the sampling channels to double. III) Abundant functions of software can realize more professional, intelligent and generic tests. IV) Sampling frequencies increases by $20 \%$, meeting the demand of monitoring dynamic random signals such as noise and vibration, on fluctuating pressure with wide frequency band.

The embedded aeroengine test system based on ARM and FPGA is successful and the prototype is manufactured and tested. It is found that the performance of the new system is promoted significantly, which shows the effectiveness and reliability of the method employed.
\end{abstract}

\section{Introduction}

Along with the development of the embedded system, because of its simplicity and specificity, the embedded system is adopted in more and more areas, especially in aeronautical and astronautically areas. But at present, foreign commercial system such as windows CE and VxWorks take a leading position, which is a potential threat to national security because the code is not open source. In the light of this disadvantage, the open source Linux system is adopted in this design, which is easy to develop and modify and has a great flexibility.

Aero-engine is the power provider of flight vehicles, which involves directly the security of flight. Therefore the improvement of maintenance and inspection level of aeroengine has become a crucial task. In recent years, although many daily testing and maintenance equipments of aeroengine based on the MCU, DSP and Industrial control computer have been developed for testing and 
maintenance. But due to low precision, single function, low intelligence, and high price, these equipments can't be equipped in large scale. The advanced embedded system can improve the quality of inspection and meet the demand of fast deployment and instant maintenance, as a result, has a more promising future.

The development of modern software and microelectronics provides good reservation for embedded technology. First, the embedded processor has smaller size, lower power consumption and richer integrated controller, faster computing speed. Secondly, the embedded microprocessor has a characteristic of abundant resources, which can transplant various embedded operating systems. So the data processing capacity of real-time operating system will be greatly enhanced, and the rich GUI interface can develop the aero-engine data testing and diagnostic system of perfect functional in order to realize the specialization of maintenance, troubleshooting and intelligence.

Linux is an operating system of open source developed by worldwide free software enthusiasts which runs in workstation and embedded device. Now the newest version is 2.6.x kernel. QT is an open source GUI development system of Sweden Nokia company. QT can provide a lot of encapsulated GUI libraries and application libraries, which enable cross platform application. QT can proceed on the system of Windows, Linux, UNIX and Mac, but also can do a compilation on multiple platform compilers which improves the transferability.

S3C2440A CPU is an industrial 400Mhz ARM920T core 16/32-bit CPU developed by Korea's Samsung company [1]. This core integrates a variety of controller, such as IIC, IIS, SPI, Flash, LCD controller etc, which has abundant resources, and convenient integration development. So the development costs can be greatly reduced.

Industrial product XC3S1600E-4FGG400I from Xilinx is selected as the Field-Programmable Gate Arrays (FPGA), which has a good performance-to-price ratio and more than 100 million integrated components.

\section{The Overall Scheme of the System}

The system consists of two parts: one is the PC data replay, storage, and engine testing configuration files, the other is data acquisition and storage system (As shown in fig.1), which consists of two parts: one is ARM processor, the other is FPGA data acquisition. The collected signal contains switching signal and analog signal, which need to be processed before FPGA. Then the collected data were processed in FPGA and stored in the dual-port RAM. These data will be read and displayed by ARM processor.

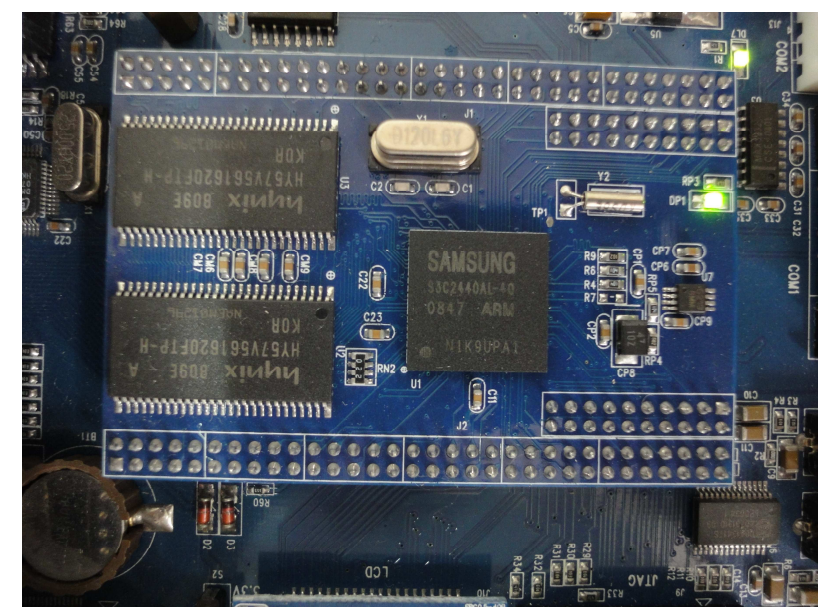

Fig. 1. Prototype of system

Software system uses Linux operating system and GUI display. Data processing adopts QT class library. After data extraction, data will be displayed in the interface and stored in the flash chip at the same time. In the S3C2440A, we use the camera controller to increase serpentine video so that it can do inspection that the eye can not reach deep inside the engine. The user can derive these data through $\mathrm{U}$ dish or CF card. The operation of software adopts the way of a touch screen to compile driver into 
kernel. At Last, in the PC, data is analyzed, replayed and stored in database. Additionally, it also needs adding to the editing function of configuration files, so as to know the change of collection and amount of acquisition range by the engine model transformation, and enhance the adaptability of the system [2].

\section{The composition of system hardware}

The data management system is managed by S3C2440A processor [3]. First, the front collected data enter the high-speed A/D converter after recuperating and proceeding and the data enters the FPGA to do data collection. After data acquisition, the data are stored in the dual-port RAM. Because the collection speed of FPGA is faster than the data extraction speed of ARM, we adopt the DMA mode for data transmission, then add the buffer pool of RAM [4]. At last, data are displayed in the ARM processor and added in the flash chip at the same time. Between the ARM processor and FPGA, connection by the dual-port RAM. It improves the stability and real time of system, and the makes the overall system perform better. The hardware design of the ARM is shown in fig 2.

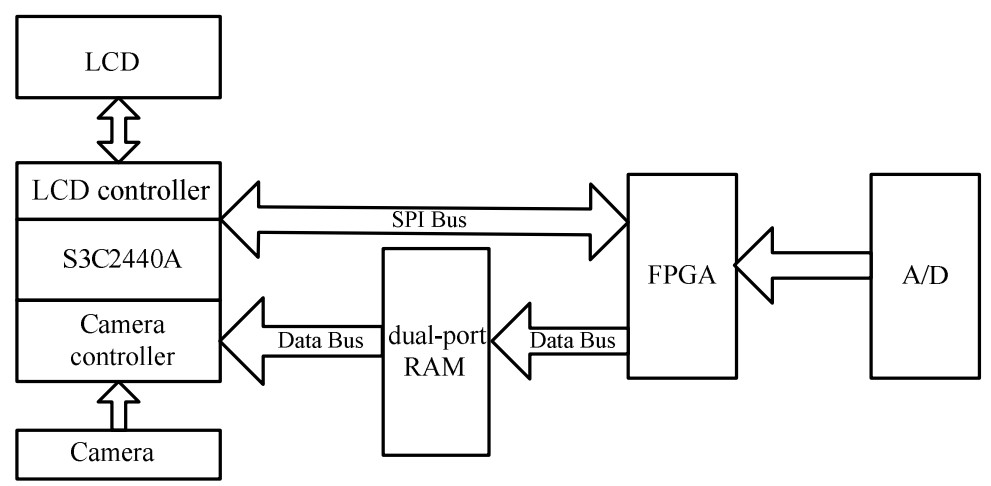

Fig. 2. The design diagram of ARM hardware

To FPGA, the design adopts the popular VHDL hardware language design. Actual running state in FPGA is simulated in the MAXPLUS II which is beneficial for accelerating the development process, the complied file flash prom into the internal of chip finally.

The clock control of A/D acquisition is provided by FPGA, which controls the change of frequency of acquisition, When the ARM starts up, acquisition parameters are written by SPI bus to FPGA. The address signal of A/D chip is provided by the FPGA. And A/D conversion chip is the core component of data acquisition system, and directly related to the performance of system [5]. We apply 12 bit successive approximation model with A/D chip AD7321 of ADI company. In the system, the chip uses the internal $2.5 \mathrm{~V}$ and the input analog reference voltage range is $0 \sim 10 \mathrm{~V}$. The chip can finish an A/D conversion in 2 us, which is because it uses the $12 \mathrm{MHz}$ clock signal that is generated by FPGA. If the interval time of two successive samples is set for 2 us, the transmission rate of system data can reach $6 \mathrm{Mps}$.

The system uses the video controller of S3C2440A to increase serpent video, and collect the video data by the micro-video chip. Of course, in the probe, the LED light is put in, so that operation personnel can conveniently better do video detection on engine blades better.

\section{Software system}

Due to the adoption of Linux operation system, the system transplant can more easily proceed, and the open of kernel code source is convenient to driver development. It exerts the better hardware efficiency and beneficial to information security. If you add your own file system, then it will be conducive to information security and prevent the leak of data in the terminal. 


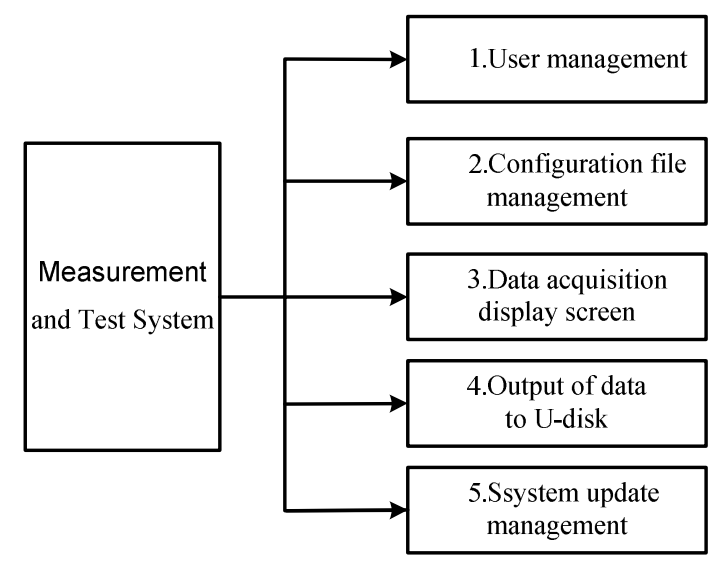

Fig. 3. The design diagram of software

Software functions are introduced concretely as follows:

1) User management

User management module is mainly to manage the user of entering the system, which has the two characters of super administrator and ordinary user. The super administrator mainly manages the ordinary user and views the login logs. The ordinary user module is mainly responsible for the function of revising the password etc.

2) Configuration files management

When configuring files, system operates the basic parameter file, the parameters are configured to the acquisition of the engine. Different engines reflect different parameters with great flexibility.

3) Data acquisition display screen

Collection interface is mainly responsible for the display of collected data, and prompt of fault state including voice and color hints.

4) Output of Data to U-disk

This module is mainly responsible for dumping the collected data stored in theflash chip, displaying data according to the filename order, and users choose the dumping file to directly copy.

5) System update management

Due to the improvement of user requirement or the existing problems of system in using, this will need to update at this time to meet users' needs. In the process of special software data storage and playback in the PC, the data is stored by encrypted file system of itself definition, so data can be stored in the database after importing PC and also can pass special network transmission to the database [6]. The designer can well know the use of engine and maintenance, to improve design better.

\section{Driver Development}

Because of the limited space, the development of the device drivers can't be discussed in detail. Only the main procedure of the development of dual-port RAM drivers is introduced. There is a big change between the new Linux 2.6 kernel core and previous Linux kernel version [7]. The following information is added to devs. c in arch/arm/mach-s3c2440/. 
The following figure are data curves acquired by the devices using applications.

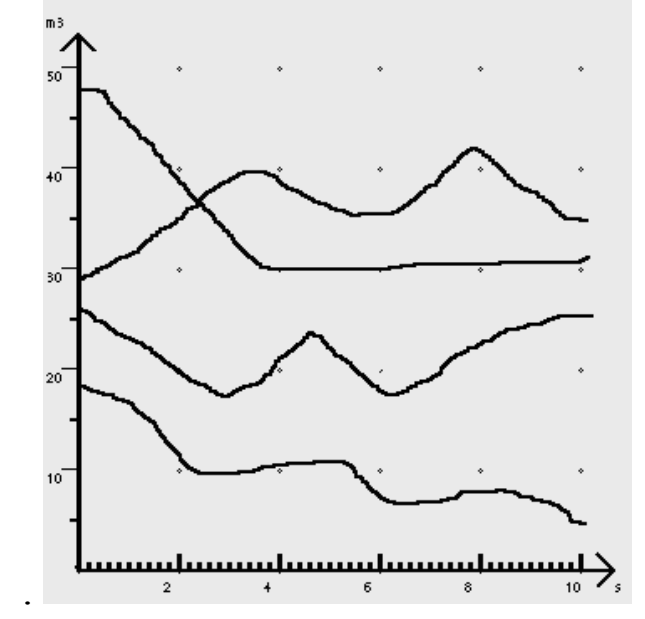

Fig. 4. The data curves acquired by the devices

\section{Conclusions}

The volume, power dissipation and cost have been greatly reduced by using S3C2440A. The design risks and cost are reduced by using the controller integrated in S3C2440A. Industrial S3C2440A can provide stable work environment, enhancing the throughput of the system greatly.

Multiple channel data sampling and tranfertation are well realized, taking the advantage of the high-speed and stable capacity of FPGA.

In on-the-spot test, Linux can run well on S3C2440A, which has realized data sampling, display and storage, achieving design targets.

Although all design demands meeted, there are still drawbacks. Linux is not a specialized real time system. Thus open sources RTEMS will be adopted in further test.

The design presented in this paper is based on the latest embedded technique, which aims to be light, elaborate, exclusive and power saving. This design takes the full advantages of ARM and FPGA, and makes reasonable arrangement for them. It provides an excellent maintenance toolkit for maintainer, which ensures the safety of flights.

\section{References}

[1] Samsung Electronics Co. S3C2440A 32 bit RISC Microprocessor User Manual (2004)

[2] S.W. Kwon, J.Y. Kim, H.S. Yoo, etc. Development of wireless vibration sensor using MEMS for tunnel construction and maintenance, J. Tunnellong and Underground Space Technology (2006) 21(3) 318

[3] A.N. Sloss, Dominic Symes, Chris Wright, ARM System Developer's Guide: Desiging and Optimizing System Software. Elsevier (2005)

[4] Jesus M. Corres, Javier Bravo, Franciso J. Arregui, etc. Vibration monitoring in electrical engines using an in-line fiber etalon, J. Sensors and Actuators A: Physical,132(2) 490-512

[5] Y.S. Fan, G.T. Zheng, Research of high-resolution vibration signal detection technique and application to mechanical fault diagnosis, J. Mechanical Systems and Signal Processing, 21(2) 610-685

[6] Studavt J D, Vinson J W, Turbome, An Expert System to Aidin the Diagnosis of Causes of Vibration Producting Problem, C. Large Turbomachinery Procedings of International Conf, Boston, USA (1985) 22 27

[7] Corbet J., Rubini A., Kroah-Hartman G., Linux Device Drivers 3th. O’Reilly (2005) 4 